\title{
Persuasive strategies in mobile insomnia therapy: alignment, adaptation, and motivational support
}

\author{
Robbert Jan Beun
}

Received: 7 November 2011/Accepted: 17 February 2012/Published online: 8 July 2012

(C) The Author(s) 2012. This article is published with open access at Springerlink.com

\begin{abstract}
The aim of this paper is to reflect on the underlying principles of insomnia therapy in relation to the use of mobile technology and to present a starting point in the design of computerized insomnia treatment. Three general persuasive strategies are presented for the design of a virtual mobile coach that functions as a first intervention for insomnia treatment in a stepped care context. The presented strategies are based on general principles and methods from cognitive behavior therapy and communication theory. In this paper, it is assumed that the motivation to perform the therapy exercises significantly can be improved by individual tailoring to the characteristics and the circumstances of the user. Two of the presented strategies aim at tailoring the intervention: alignment and adaptation; the third strategy, motivational support, aims at the coach's communicative activities to take away motivational blockades. Given the characteristics of the insomnia treatment, it is concluded that mobile and ubiquitous systems are promising monitoring and delivery devices in a first intervention, provided that the information is reliable and that obtrusiveness is minimized.
\end{abstract}

Keywords Adaptation $\cdot$ Alignment $\cdot$ Motivational support $\cdot$ Insomnia $\cdot$ Mobile coaching $\cdot$ Persuasion

\section{Introduction}

Probably, one of the most well-known systems developed to trigger people to change their activity is the alarm clock

R. J. Beun $(\bowtie)$

Information and Computing Sciences, Utrecht University, PO Box 80.089, 3508 TB Utrecht, The Netherlands

e-mail: r.j.beun@uu.nl that wakes us up in morning. The alarm clock can be considered as a persuasive tool: it helps the user to change a state of sleep into a state of wakefulness and reminds him or her to get up from the bed. For most people, the alarm clock works fine, but for those who suffer from sleep disorders, such as insomnia, it hardly gives the desired or necessary support to cope with the sleep problem or with the daily discomfort that follows from this problem.

The aim of this paper is to present some general persuasive strategies to be used by a virtual mobile coach in the field of self-help insomnia treatment. In particular, we will focus on strategies grounded in cognitive behavior therapy (CBT) and communication theory, and show the potential of mobile and ubiquitous technology in this treatment.

Self-help CBT for insomnia (CBT-I) has been proposed as a first option in stepped care context. In a stepped care context, an individual first receives the least restrictive treatment that is still likely to provide significant health gain. These therapies are targeted at large user groups, easy accessible, and cost-effective [13, 23, 42]. As we will see, current self-help therapies from books and television improve coping with insomnia, but they often do not lead to sleep improvements. An important aspect missing from many self-help therapies is the essential personalized support to encourage people to adhere to the therapy.

In this paper, two important assumptions will be made. First, motivation and, consequently, treatment efficacy of current self-help interventions can significantly be improved with (semi-)automated and individually tailored programs. Second, the therapy should take a holistic approach, meaning that the coachee ${ }^{1}$ should be considered

\footnotetext{
"We will use the term "coachee" or "person" for the one who follows the therapy, instead of, for instance, "client," "patient," or "user".
} 
as an individual with daily physical, mental, and social activities in a complex physical and networked environment with other individuals. This connects to the emerging interest in developing automated mobile systems that can provide health services to patients and consumers without direct human interference $[6,7]$. In these systems, methods from persuasive technology are applied to implement effective communication strategies that support self-care and adherence to prescribed treatments and exercises $[14,15,18]$.

Let us, before we discuss the adherence problem of CBT-I and some possible solutions in terms of the presented strategies and delivery methods, first present some relevant characteristics of insomnia and insomnia therapy.

\section{Insomnia}

Sleep disorders, such as insomnia, are among the most prevalent mental disorders in the general population. The working definition of insomnia is "a persistent difficulty initiating and/or maintaining sleep" [26]. Insomnia has an enormous impact on the individual and society. It impairs daily functioning, reduces quality of life, and enhances health care and other costs for millions worldwide. Measurable costs of insomnia include reduced productivity, increased absenteeism, accidents, and hospitalization, as well as medical costs due to increased morbidity and mortality, depression, and increased alcohol consumption [11]. Already, in 1996 Roth [35] estimated the overall economic costs of insomnia in the United States at about 30-35 billion dollar.

Insomnia can usually be discerned from clinical interview and shows a large variability of individual differences in both causes and symptoms [20]. In some individuals, insomnia is caused by their lifestyle (e.g., irregular times for sleeping, lack of exercise and relaxation, alcohol and drugs abuse, and anxiety); in others, psychiatric or medical disturbances cause insomnia (e.g., depression, stress, asthma, and pain). The underlying mechanisms are still poorly understood and in many cases, the reason for the disorder is unknown.

For chronic insomnia, complaints have to stay for at least a month [3]. Chronic insomnia is a common sleep disorder with a prevalence of around $10 \%$ in the general population $[2,28]$.

\subsection{Insomnia treatment}

Generally, the treatment for insomnia entails non-pharmacologic and/or pharmacologic aspects. If the insomnia has lasted only a few days or weeks, hypnotics may be the treatment of choice. Long-term treatment with hypnotics, however, causes dependence, side effects on daily functioning, disorganized sleep architecture, and the chance of rebound insomnia after withdrawal [26].

Today, it is widely accepted that cognitive and behavioral factors play an important role in the condition of insomnia and that non-pharmacological treatments such as CBT-I produce sustainable positive changes in this condition $[12,21,22,25,28]$. CBT-I is designed to change dysfunctional beliefs, attitudes, and behavior that support sleep-disruptive habits, thoughts, and emotions.

CBT-I usually rests on two pillars: (1) a behavioral component to unlearn maladaptive habits and to learn sleep improving behavior and (2) a cognitive component to change a person's dysfunctional cognition, such as negative thinking or unrealistic expectations with respect to poor sleep. Treatment protocols for insomnia usually take between 6 and 10 weekly consultation sessions [25]. Depending on the stage of the therapy, various activities may be scheduled during a session: for instance, presentation of a conceptual model of insomnia, review of the person's sleep diary, explanation of clinical procedures and their rational, evaluation of compliance with homework assignments, and introduction of new assignments for the upcoming week.

Basically, CBT-I offers a number of exercise types whose effectiveness is proven for sleep therapy that is, sleep restriction, stimulus control, relaxation, and sleep hygiene [21, 25, 26, 28, 29, 43]. Sleep restriction involves curtailing the time spent in bed to stabilize the sleep pattern and then lengthening sleep time as sleep efficiency improves. Stimulus control is aimed at the coachee's reassociation of the bed and the bedroom with sleep and to re-establish a consistent sleep-wake schedule; consequently, a person should avoid waking activities in bed, such as worrying, reading, eating, and watching television (sexual activity excepted), and, vice versa, a person should avoid sleeping activity at locations other than the bed, for instance, in front of the television. Relaxation training involves methods aimed at reducing somatic tension. Sleep hygiene education aims to make the person aware of health practices and environmental factors that may either be detrimental or beneficial for sleep. Since sleep restriction and stimulus control show an obvious synergy, they can be combined in the more general term "sleep scheduling".

\section{Delivery methods and technology for CBT-I}

A variety of delivery methods is described with respect to CBT-I: individual sessions [26], group sessions [4], selfhelp approaches by books, audio and video [23], and the use of ICT such as telephone and Internet consultation, and television [30,43]. A commercial Dutch Internet therapy is provided by Somnio (www.somnio.nl). 
Unfortunately, CBT-I has some critical shortcomings. Weekly face-to-face sessions with human therapists are expensive and intense involvement of health care professionals results in long waiting lists. Although self-help therapies from books and television improve coping with insomnia, these therapies often do not lead to sleep improvements.

In a recent meta-analysis, the effects of self-help for insomnia were found to be small to moderate [42], but Internet-delivered trials have been published with more promising results [34, 45]. Recently, a large-scale Dutch trial was completed where positive effectiveness was found on sleep measures [22].

In the area of sleep technology, three types of consumer products can be distinguished: (a) physical products that change the physical environment or the user's perception of the environment, like ear plugs and light and sound changing devices, (b) lifestyle products aimed at change of behavior and cognition, (c) specialized products such as masks for sleep apnea. Today, a multitude of electronic consumer products exists that are targeted toward sleeprelated inconveniences, such as Philips' Wake-up Light, the aXbo wristband and Audio Rider's sleep mattress "Carerider".

A recent development that opens a way for automated individualized sleep support is the availability of computerized consumer products, such as Zeo Personal Sleep Coach (www.myzeo.com), the handheld computer device Sleepkey ([32], www.sleepkey.com), and many small applications available in, for instance, Apple's iPhone store. A categorization of this type of technology is presented in [9] where three themes are identified: (a) sleep tracking, (b) waking and sleep inducing, and (c) social applications. Sleep tracking is directed at the observation of individual sleep characteristics, such as frequency, duration, or quality of sleep; tracking can be done by automatic sensing, manual input, or both. Waking and sleep-inducing technology supports people to wake up in the morning or go to sleep at night. Social applications focus on social aspects of sleep, such as the sharing of sleep status within a social network as a tool for awareness. In general, a major problem is that a proliferation of sleep applications arises regardless of real user needs and that hardly any treatment or technique of these products is evidence-based [9].

The lack of evidence for the effectiveness of self-help therapies does not imply that self-help in the domain of insomnia cannot work in the future. An important problem with current self-help interventions, such as books and Web sites, is that the therapy is targeted at large user groups and does not take the individual characteristics of users into account. Another problem is that CBT-I usually requires strong motivation and discipline of the coachee and that adherence to the therapy is difficult without tailored exercises or mental support. In other words, personalization plays a key role in the therapy. The need for personalized information is met by the success of the Internet-delivered therapies where human coaches are still part of the therapy (e.g., [20]). In principle, computerized CBT-I enables us to design and implement individually tailored programs, but the launch of these programs into the market should be preceded by careful analysis of the coaching process. CBT-I performed by human coaches consists of many different elements, ranging from a clear understanding by the coach of the problem to the timing of relevant messages and building the coachee's confidence about the proposed exercises. As a consequence, computerized CBT-I requires understanding of the problem at many different levels in order to work properly. The aim of this paper is, therefore, to present a starting point in the design of computerized CBT-I and to reflect on the underlying principles of CBT-I in relation to the presented persuasive strategies and the potential possibilities offered by mobile technology.

\section{Therapy compliance and motivation}

In general, one of the major problems in CBT is noncompliance (or non-adherence) to the therapy (c.f. [6]) and CBT-I is no exception to this. In CBT-I, some exercises demand extensive self-discipline and stamina of the coachee. People may find the exercises too strenuous (e.g., "sleep restriction"), they do not believe that it contributes to a solution of the problem (e.g., a relaxation exercise) or simply forget to perform the exercise (e.g., filling in their sleep diary).

One of the keys to therapy adherence is the person's motivation to perform the exercises. People may enthusiastically start a particular self-help sleep therapy and discover that, as part of a sleep restriction exercise, reducing their time spent in bed or getting out of bed in the middle of a cold and dark night requires a great deal of effort. Consequently, sleep quality deteriorates, people feel worse, get into a downward motivation spiral, and the therapy is terminated prematurely. Recognition of this process and adequately responding to it in terms of, for instance, motivational support is a necessary element in any insomnia therapy. Therefore, as any other computerized CBT selfhelp system, a mobile CBT-I system has to include a variety of persuasive elements and strategies to improve adherence to the therapy. To understand the value of these strategies, let us first explore the concept of motivation (c.f. [16]). 


\subsection{Motivation, activities, and goals}

Motivation research has a long history in psychological science, and definitions are not widely shared among researchers. Roughly, to be motivated means to be moved to do something [36]; motivation gives direction to people's activities. In formal communication and agent theories, motivation is often expressed in mental constructs such as wants, desires, obligations, or intentions to achieve a particular state, the so-called goal; these constructs are essential parts of the preconditions to generate speech acts or other activities [5]. Goals in insomnia treatment can be, for instance, improved sleep quality and daytime functioning, increased sleep efficiency, and reduced time to fall asleep. Activities are the exercises to achieve these goals.

In traditional motivation theories [36], different types of activities are distinguished: activities may be intrinsically or extrinsically motivated. Intrinsically motivated activities, or briefly "intrinsic activities," refer to activities in which someone experiences a reward in the activity itself, a task that one "wants" to do. A hungry person, for instance, experiences a reward in the activity of eating. Extrinsic activities refer to activities for which there is no inherent connection between the activity and the reward, a task that one "has" to do for whatever reason. The reward in the extrinsic activity is in the expected goal; the person has to invest a certain amount of energy and time, and expects that payback will happen once this goal has been achieved. A hungry person that seeks for food believes that the activity of seeking will bring the person in a rewarding situation when the food is found and can be eaten. Unfortunately, sleep therapy requires many extrinsic activities, in particular in the beginning, which makes the therapy design highly complex. One of the most demanding extrinsic actions is part of the sleep scheduling exercise, namely getting up if unable to sleep quickly.

In line with the two types of activities, we also distinguish between goals that are intrinsic and goals that are extrinsic. An intrinsic goal refers to a state in which one experiences a reward simply by achieving the goal. Extrinsic goals refer to a state where one does not or hardly experiences a reward, a necessary and rationalized intermediate state to achieve the intrinsic goal. For instance, the overall outcome of CBT-I is an intrinsic goal, because improved quality of sleep implies that the person feels better after the therapy. Goals such as increased sleep efficiency are extrinsic, imposed by the coach and do not necessarily lead to an improved feeling of well-being. In fact, improvement of sleep efficiency usually decreases the feeling of well-being, because curtailing the time spent in bed may cause a constant feeling of drowsiness during the day.
In practice, however, the distinction is not always that clear. A particular activity may be assigned a particular value and may vary over time and individual - an option would be, for instance, to assign extrinsic activities with negative numbers ("punishment") and intrinsic with positive numbers ("pleasure"). In CBT-I, a relaxation exercise may be helpful for some individuals, but may be counterproductive for another. What is important, though, is that intrinsic activities are rewarding in itself and can be used as a strategy to improve motivation and that extrinsic activities and goals need more effort from the coach in terms of persuasion techniques. Intrinsic activities need only brief support, a simple trigger may be sufficient. Extrinsic activities may call for detailed explanation with respect to their purpose, expected investments, or effects.

The notions of "trust" and "credibility" play a particular role in extrinsic activities and goals. A person performing extrinsic activities loses sense for direction and leaves the decision to perform a particular exercise to the coach. A person who does not trust the coach and, therefore, does not believe that an exercise is in the person's best interest can hardly be motivated to perform the exercise.

Motivation is not an invariant mental construct that remains constant during the therapy and may dramatically decrease as long as the reward is delayed. In fact, as we discussed earlier, loss of motivation is one of the key factors in aborting the therapy. Its baseline value in the beginning of the therapy is influenced by many factors, such as the initial attitude, stage of change [31], personality, beliefs, moods, expectations, progress, current interests, circumstances, emotions, and so on.

\section{Persuasive strategies in CBT-I}

Assuming that motivation plays a crucial role in therapy adherence, the previous discussion raises some important questions: for instance, how and when should motivation be monitored, how can loss of motivation be minimized, should the coach adapt to a particular level and respond to the variations and if so, how? Clearly, we have no definite answers to these questions, but in the remainder of this paper, we will explore the three persuasive strategies that may influence motivation considerably in a positive manner: "alignment," "adaptation," and "motivational support". Alignment enables us to establish familiarity between coach and coachee, and to determine a motivational baseline; adaptation is a means of adjusting exercises and information exchange to individual needs, abilities, and knowledge; motivational support is a communicative activity that is geared toward the removal of physical and 
mental motivational blockades. Alignment may be taken as a prerequisite for both adaptation and motivational support.

\subsection{Alignment}

On an abstract level, CBT-I can be considered as an amalgam of individual and joint activities [10]. Coachees carry out individual exercises suggested by the coach, and there is frequent coordination of physical and communicative activity between coach and coachee, in particular in those cases where the coach will be presented by a mobile device that takes part in the coachee's daily life.

To avoid unnecessary loss in motivation, the coach should minimize disturbance of the coachee's daily activities. Interruptions, for instance, are the basic events where the activities of the coach and coachee meet. They may be considered as signals by the sender indicating that the intended receiver should shift the attention toward another activity. Some interruptions are more welcome than others, however. A person that phones during a meditation activity of another person to return the wallet that the meditator just lost is usually highly welcome. But, depending on the current activity and the time schedule for the next $2 \mathrm{~h}$, the unexpected phone call of a fellow bad sleeper may be welcome or unwelcome. And a system that randomly generates auditory signals that wake someone up in the middle of the night is probably always unwelcome.

In sleep therapy, intervention is sometimes inevitable and requires careful interaction, but superfluous interruptions and irrelevant interaction have to be avoided at all costs. A virtual mobile coach should, therefore, become familiar with a blend of characteristics of the coachee: the current activity, the schedule, the attitude toward particular exercises, the ability to perform the exercise, and so on. In other words, coordination of activities requires strong awareness of the characteristics and activities of the coachee and the relevant features of the situation at hand.

A sine qua none for joint activities, and conversation in particular, is "common ground," that is, a common understanding of ourselves, our communication partners, and the world around us. To achieve common ground, coach and coachee need to align their situation models, the so-called process of alignment [17]. Since alignment is a two-way process, the coachee should get acquainted with relevant characteristics of the coach as well. Incorporating alignment skills in mobile CBT-I is a prerequisite for tailoring information and minimizes the exchange of irrelevant and superfluous information.

In human conversation, alignment is largely an unconscious process where participants align their representations at various levels. Alignment with a virtual coach may refer to at least three levels of interaction:
- the therapy level, that is, information that is used to offer an adequate individualized therapy. Examples are the coachee's state of change, diagnosis of the sleep problem (e.g., $[8,38]$ ), age, expectations and investments, sleep and wake habits, time schedules, former therapies, mutual commitments, therapy starting point and program, system role, and insomnia-related knowledge.

- the communication level, that is, information that refers to the interaction itself between coach and coachee. Examples are interface design, formality, politeness, personal reference, gender, modality preference, and language use.

- the ethical level, that is, information that refers to the interests of the coach and coachee to establish a relation of trust. Examples are privacy and safety-related matters, the involvement of other parties, the system's credibility, commercial interests, and risks of the therapy.

A powerful alignment strategy is the mutual acceptation of the therapy goals and commitments [39]. For instance, coach and coachee may agree upon $1 \mathrm{~h}$ exercising a day or a sleep efficiency of $85 \%$ as a goal. Reminding the coachee of these goals and commitments may be considered as an example of motivational support (see below).

Since alignment plays a crucial rule in all types of interaction, we recommend in line with human therapy the inclusion of an explicit stage in the therapy model where coach and coachee learn to know each other; in sleep therapy, this stage should take about a week from the start (c.f. [27]). It should be kept in mind, however, that alignment is a constant process throughout the therapy and that it requires frequent feedback.

A major problem in mobile applications is that the capacity of the information channel is low compared to a human coach. As a consequence, the interface and the dialogue should be carefully designed and the communication should be tailored as much as possible to the characteristics of the coachee (see below). On the other hand, because a mobile application is in principle always present in the environment of the coachee, it enables interaction whenever needed.

\subsection{Adaptation}

As in any other type of joint activities, human coaches in CBT-I perform intelligent and cooperative behavior to achieve the goals of the activities. One of the basic components of these types of behavior is adaptation [40]. By continuous monitoring and feedback (alignment), human participants in conversation are able to adapt constantly to their environment and communication partners. Tailoring, 
personalization, and customization may be considered as special cases of adaptation. Here, adaptation will be limited to the process of adjusting the information exchange and sleep exercises to the characteristics and the circumstances of coachees, such as their needs, abilities, preferences, knowledge, and location. In all cases, a user model is a necessary requirement.

\subsubsection{Information exchange}

To adapt the communicative actions performed by the agent, we may find important inspiration in the so-called Gricean maxims [19]. These maxims roughly state that in cooperative settings, conversational participants should not lie (quality maxim), should give neither too much and nor too little information (quantity), that information should be relevant (relevance), and should be performed in the right manner (manner). Note the time dependency of the relevance maxim; what is relevant at a specific point in time does not have to be relevant at another.

Although originally focused on the explanation of a particular type of conversational inferences (so-called conversational implicatures), the maxims form a powerful intuitive framework for the design of conversation and can effortlessly be transferred to the domain of sleep coaching. For instance, argumentation skills could be built in to solve conflicting information between objective and subjective sleep tracking (quality), sleep-related information that was already communicated could be left out or should only be used in a presuppositional position (quantity), information should only be exchanged with respect to the accepted goals of the therapy (relevance), and depending on the coachee's preferences, the interaction language and its modality could be adapted to a particular level of politeness, literacy of the coachee, or the level of noise in the environment (manner).

\subsubsection{Exercising}

As we discussed before, an important problem is that there is constant investment in the first 3 or 4 weeks of an insomnia therapy without any payback. Even worse, in the beginning, people feel that they drift away from the overall goal of the therapy. Sleep efficiency may decrease, sleep quality and daily functioning may deteriorate, and the light at the end of the tunnel seems unreachable without any help. There are at least two ways to reduce this tension: adaptation of the exercises and/or adaptation of the goal(s). Let us briefly discuss the first.

Often, there is room for negotiating some of the exercise variables such as timing, duration, and effort. On the one hand, sleep therapy requires discipline and, therefore, some extrinsic exercises are mandatory and just have to be done
[27]. On the other hand, there is room for the adaptation of the exercise time schedule. For instance, on the basis of an initial period of alignment, the coach suggests a particular threshold time to go to bed and rising time to get up. Within limits, individual preferences may be incorporated in the actual schedule to indicate a delay or advance in rising and threshold time suggested by the system. Also, some exercises may work better than others; therefore, substitution of comparable exercises may be an option in some cases. Substitution may work in particular for relaxation exercises. In other words, there should be room for exercise adaptation wherever possible. Because exercise adaptation can only be done within limits, a mobile system should be endowed with communication capabilities such as negotiation and explanation.

\subsection{Motivational support}

Motivational support refers to verbal and/or nonverbal communication acts to take away physical and mental motivational blockades. It may vary from a single reminder to start the exercise to an explicit compliment or feedback related to the coachee's progression. Both adaptation and alignment are prerequisites for the generation of effective motivational support.

Motivational support relates partly to the cognitive part of CBT-I. Cognitive therapy is a psychotherapeutic method designed to change a person's beliefs, expectations, appraisals, and attributions. In CBT-I, cognitive therapy seeks to change dysfunctional sleep expectations, perceived causes and consequences of insomnia, and beliefs about sleep-promoting practices [27]. Assume, for instance, a person that believes that one night bad sleep always causes poor work performance. As a result of this prospect, motivation decreases. The primary goal of the coach would be to support the coachee to re-evaluate the accuracy of this belief.

Other types of motivational support may be, for instance, encouragement, challenge, awareness of progress and self-responsibility, showing or telling consequences of behavior, showing discrepancy in present behavior and mutual goal commitment, support of self-efficacy, and rewards such as statements of appreciation. A major challenge for mobile applications is how the timing and the content of this type of support are related to the coachee's daily activities, performances, and characteristics.

\section{Ubiquity and mobile devices in CBT-I}

The strength of a virtual coach delivered by a mobile device lies in the constant access to the attention space of the user. The device not only enables the coach to deliver 
messages in time, but also to assess momentary information by questioning the user or by monitoring the physical surrounding and biosignals from the user (see also [37]). Vice versa, the user has constant access to the attention space of the coach. Whenever desirable or needed, the user may indicate the readiness to start a particular exercise or ask for motivational support, for instance, questions about the relevance of the exercises, information about the progress, or any other kind of help.

As a consequence, the concept of ubiquity and the accompanying sensor technology will play an important role in both the adaptation of the sleep therapy and the motivational support. Information about environmental conditions is relevant in all types of exercises and stages of the therapy. Biosignals such as heart rate variability, EEG, muscle tension, skin conductance, movement, and respiration give important information about the degree of relaxation, and about sleep and sleep stages [44, 46]. In the future, automated snoring detection may be of great value in the alignment phase. In sleep hygiene, we may think of monitoring temperature, sound, and light conditions of the bedroom.

Not only is ubiquity needed in the adaptation of exercises, it also enables the system to determine an optimal moment for interruption and to adapt its communication. Data about the coachee's location, in terms of bedroom, living room, or office, may provide information about the coachee's activities and may support the choice of a particular hardware device for interaction. Surrounding conditions (e.g., light and dark) may determine the choice and characteristics of the communication modality in terms of visual or auditory signaling or brightness and loudness.

From the previous, it may be concluded that mobility and ubiquity are beyond doubt. It should be noted, though, that integrity of the data processing and reliable sensing is an important prerequisite for acceptation of the system. Also, intrusive measurements should be avoided wherever possible in a first-step therapy. Consequently, a trade-off should be found between reliability, intrusiveness, and integrity of the information. For instance, reliable sensing of sleep and sleep stages is a complex endeavor that needs highly intrusive sensing methods such as polysomnography. ${ }^{2}$ A major challenge is, therefore, the development of non-obtrusive sensing technology from which sound

\footnotetext{
${ }^{2}$ Polysomnography records various biophysical changes that occur during sleep, such as eye movements, brain waves, and heart rhythm and is used to diagnose various types of sleep characteristics and sleep disorders. The method is highly obtrusive, because a minimum of 22 wires are attached to the patient; it is, therefore, often only used in a sleep laboratory. A polysomnographic test may yield information about, for instance, sleep stages, sleep efficiency, and breathing irregularities. It is considered as the golden standard in sleep measurement.
}

inferences can be drawn with respect to automated sleep tracking.

This brings us to some technical details with respect to the architecture of these types of systems. At a particular level of information processing of the virtual coach, two types of information flow with the outside world have to be integrated: (a) symbolic, such as linguistic and iconic data from sleep diary or dialogue, and (b) sensory, such as body movement or light conditions (c.f. [1]). Because sensor data may come from heterogeneous hardware devices, the system has to account for the integration of these data at a meaningful ontological level. Location data available from Bluetooth and GPS, for instance, have to be translated into therapy-relevant concepts such as "bedroom," "living room," or "away from home". In many cases, a single sensor will not provide enough information and, consequently, a fusion across sensors, attributes, and domains will be necessary to improve the quality of information [24]. As a first step, we may think of the multi-agent paradigm such as developed in [41]. In this agent model, a layered structure of ontologies and communication strategies is applied to achieve semantic interoperability between various types of information systems (c.f. [44] for sleeprelated data).

\section{Conclusion}

In this paper, we have outlined some fundamental strategies for a mobile CBT-I coach to improve therapy adherence. Compared to traditional human-human therapy and self-help books, mobile and ubiquitous devices have a number of important advantages. First, the use of mobile technology permits the assessment of relevant momentary information and the delivery of fully automated feedback in the appropriate form and modality, at the appropriate time and location. Second, the existing technology enables tailored treatment on the basis of both personalized and environmental information of the individual user. Third, non-obtrusive sensory measurement enables the obtainment of objective sleep data that can be integrated in the treatment. Fourth, current communication platforms and infrastructures facilitate information exchange between various user groups, such as peer insomniacs, sleep therapists, and sleep expert centers; in other words, these systems can be part of a stepped health care structure where care providers only are involved when problems are detected.

In relation to the last point, it is important to keep in mind that a large group of insomniacs will still need professional assistance provided by individual sleep therapists or sleeping disorder centers. In these cases, a virtual coach may give valuable support for both the patient and the 
therapist. It may provide for rich data concerning the patient's sleep and wake behavior before the actual treatment begins. Hence, the diagnosis process does not solely depend on data from the laboratory (e.g., polysomnographic data), but also from data that shows the dynamics of sleep and wake state in the patient's daily environment. Moreover, during the treatment, the virtual coach may also provide for techniques to support the exercises and the evaluation process of the patient's compliance with the clinical procedures and homework assignments.

Given the many negative consequences of pharmacologics and the success of CBT-I, it can be expected that information technology, non-obtrusive sensor data, and environmental changing devices (light, sound, and temperature) will play a prominent role in a first-step therapy. For that, sleeping well should not only be considered as a health care challenge, addressing issues such as apnea, restless legs, and chronic insomnia, but also as a lifestyle challenge addressing a large consumer population that faces issues like winding down and relaxation before sleep.

Finally, the proposed persuasive strategies presented in this paper not only pertain to the area of sleep, but may be applied to other domains of persuasion as well. Our view of the future is one where virtual lifestyle and health assistance is a standard technology incorporated in various types of lifestyle products. To improve usability, devices will be accompanied by virtual cooperative agents and smoothly integrated in daily life. This opens a way for extreme customization of consumer products: use and explanation on a personal basis. In medical applications, virtual agents combined with sensors may function as sophisticated interactive manuals that support patients or elderly users with electronic health records, telemedicine services, and portable monitoring and advise systems. In education and training environments, virtual agents will be able to support tailored training programs, explain reasons for exercises and decisions, motivate students, and adapt communication to the knowledge, experience, and abilities of the user.

In the future, our alarm clock will be transformed into an intelligent and cooperative partner that smoothly adapts to its user and the circumstances of the interaction (c.f. [33]). But before this captivating prospective becomes reality, an enormous body of challenging research has to be carried out and integrated in a variety of disciplines, ranging from computing sciences and artificial intelligence to neurobiology, psychology, and communication sciences.

Acknowledgments I thank the anonymous reviewers for their helpful comments. Also, many thanks to Gaby Jankowiak, Rogier van Eijk, Sandor Spruit, Willem-Paul Brinkman, Reinder Haakma, Ingrid Verbeek, Linda Bronts, Jaap Lancee, Marjolijn Sorbi, and all the others who inspired me in the field of coaching, sleep, and mobile persuasive technology. This research is supported by Philips and
Technology Foundation STW, Nationaal Initiatief Hersenen en Cognitie NIHC under the Partnership programme Healthy Lifestyle Solutions.

Open Access This article is distributed under the terms of the Creative Commons Attribution License which permits any use, distribution, and reproduction in any medium, provided the original author(s) and the source are credited.

\section{References}

1. Ahn R, Beun R, Borghuis T, Bunt H, van Overveld C (1995) The DenK-architecture: a fundamental approach to user-interfaces. Artif Intell Rev 8(9):431-445

2. Ancoli-Israel S, Roth T (1999) Characteristics of insomnia in the United States: results of the 1991 National Sleep Foundation Survey I. Sleep 22(Suppl. 2):347-353

3. American Psychiatric Association (2000) Diagnostic and statistic manual of mental disorders, 4th edn. American Psychiatric Press, Washington

4. Backhaus J, Hohagen F, Voderholzer U, Riemann D (2001) Long-term effectiveness of a short-term cognitive-behavioral group treatment for primary insomnia. Eur Arch Psychiatry Clin Neurosci 251:35-41

5. Beun RJ (2001) On the generation of coherent dialogue: a computational approach. Pragmat Cogn 9(1):37-68

6. Bickmore T, Mauer D, Crespo F, Brown T (2007) Persuasion, task interruption and health regimen adherence. In: de Kort Y, IJsselsteijn W, Midden C, Eggen B, Fogg BJ (eds) Persuasive technology. LNCS volume 4744. Springer, Berlin, pp 1-11

7. Blanson Henkemans OA (2009) ePartner for selfcare. How to enhance eHealth with personal computer assistants. Dissertation, Technische Universiteit Delft

8. Buysse DJ, Ancoli-Israel S, Edinger JD, Lichstein KL, Morin CM (2006) Recommendations for a standard research assessment of insomnia. Sleep 29(9):1155-1173

9. Choe EK, Consolvo S, Watson NF, Kientz JA (2011) Opportunities for computing technologies to support healthy sleep behaviours. In: Proceedings of CHI 2011, 7-11 May, Vancouver, BC, Canada, pp 3053-3062

10. Clark HH (1996) Using language. Cambridge University Press, New York

11. Daley M, Charles M, Morin CM, LeBlanc M, Grégoire J-P, Savard J (2009) The economic burden of insomnia: direct and indirect costs for individuals with insomnia syndrome, insomnia symptoms, and good sleepers. Sleep 32(1):55-64

12. Edinger JD, Means MK (2005) Cognitive behavioral therapy for primary insomnia. Clin Psychol Rev 25:539-558

13. Espie CA (2009) 'Stepped care': a health technology solution for delivering cognitive behavioral therapy as a first line insomnia treatment. Sleep 32(12):1549-1558

14. Fogg BJ (2003) Persuasive technology: using computers to change what we think and do. Morgan Kaufmann Publishers, Amsterdam

15. Fogg BJ, Eckles D (eds) (2007) Mobile persuasion: 20 perspectives on the future of behavior change. Stanford Captology Media, Stanford

16. Fogg BJ (2009) A behavioural model for persuasive design. Persuasive '09, 26-29 Apr, Claremont, California

17. Garrod S, Pickering MJ (2004) Why is conversation so easy? Trends Cogn Sci 8(1):8-11

18. Gasser R, Brodbeck D, Degen M, Luthiger J, Wyss R, Reichlin S (2006) Persuasiveness of a mobile lifestyle coaching application 
using social facilitation. In: IJsselsteijn W, de Kort Y, Midden C, Eggen B, van den Hoven E (eds) Persuasive technology for human well-being: setting the scene. LNCS volume 3962/2006. Springer, Berlin, pp 27-38

19. Grice HP (1975) Logic and conversation. In: Cole P, Morgan J (eds) Syntax and semantics, vol. 3, speech acts. Academic Press, New York, pp 41-58

20. Hofman WF, Kumar A (2010) Online treatment of insomnia using behavioral techniques. Sleep Diagn Ther 5(3):28-32

21. Irwin MR, Cole JC, Nicassio PM (2006) Comparative metaanalysis of behavioural interventions for insomnia and their efficacy in middle-aged adults and in older adults 55+ years of age. Health Psychol 25(1):3-14

22. Lancee $\mathbf{J}$ (2011) Cognitive-behavioral self help treatment for nightmares and insomnia. Dissertation, Utrecht University

23. Mimeault V, Morin CM (1999) Self-help treatment for insomnia: bibliotherapy with and without professional guidance. J Consult Clin Psychol 67:511-519

24. Mitchell HB (2010) Multi-sensor data fusion. Springer, Heidelberg

25. Morgenthaler T, Kramer M, Alessi C, Friedman L, Boehlecke B, Brown T, Coleman J, Kapur V, Lee-Chiong T, Owens J, Pancer J, Swick T (2006) Practice parameters for the psychological and behavioral treatment of insomnia: an update. An American Academy of Sleep Medicine report. Sleep 29(11):1415-1419

26. Morin CM (1993) Insomnia: psychological assessment and management. Guilford Press, New York

27. Morin C, Espie C (2004) Insomnia, a clinical guide to assessment and treatment. Springer, New York

28. Morin CM, LeBlanc M, Daley M, Gregoire JP, Merette C (2006) Epidemiology of insomnia: prevalence, self-help treatments, consultations, and determinants of help-seeking behaviors. Sleep Med 7(2):123-130

29. Murtagh D, Greenwood K (1995) Identifying effective psychological treatments for insomnia: a meta analysis. J Consult Clin Psychol 63(1):79-89

30. Oosterhuis A, Klip EC (1997) The treatment of insomnia through mass media, the results of a televised behavioural training programme. Soc Sci Med 45(8):1223-1229

31. Prochaska JO, Norcross JC (2001) Stages of change. Psychotherapy 38(4):443-448

32. Riley WT, Mihm P, Behar A, Morin CM (2010) A computer device to deliver behavioral interventions for insomnia. Behav Sleep Med 8:2-15
33. Rist T, André E, Baldes S, Gebhard P, Klesen M, Kipp M, Rist P, Schmitt M (2003) A review on the development of embodied presentation agents and their application fields. In: Prendinger $\mathrm{H}$, Ishizuka M (eds) Life-like characters. Tools, affective functions and applications. Cognitive technologies series. Springer, Berlin, pp 377-404

34. Ritterband LM, Thorndike FP, Gonder-Frederick LA, Magree JC, Bailey ET, Saylor DK (2009) Efficacy of an internet-based behavioral intervention for adults with insomnia. Arch Gen Psychiatry 66(7):692-698

35. Roth T (1996) Social and economic consequences of sleep disorders. Sleep 19(8):546-547

36. Ryan RM, Deci EL (2000) Intrinsic and extrinsic motivations: classic definitions and new directions. Contemp Educ Psychol 25:54-67

37. Sorbi MJ, Rüddel H, Bühring MEF (eds) (2007) Frontiers in stepped eCare. eHealth methods in behavioural and psychosomatic medicine. A-D Druk BV, Utrecht

38. Spoormaker VI, Verbeek I, Van den Bout J, Klip EC (2005) Initial validation of the sleep-50 questionnaire. Behav Sleep Med 3(4):227-246

39. Starr J (2008) The coaching manual. The definitive guide to the process, principles and skills of personal coaching. Pearson Prentice Hall Business, London

40. Sternberg RJ (1985) Beyond IQ: a triarchic theory of human intelligence. Cambridge University Press, Cambridge

41. Van Diggelen J, Beun RJ, van Eijk RM, Werkhoven PJ (2010) Efficient semantic information exchange for ambient intelligence. Comput J 53(8):1138-1151

42. Van Straten A, Cijpers P, Smit F, Spermon M, Verbeek I (2009) Self-help treatment for insomnia through television and book: a randomized trial. Patient Educ Couns 74(1):29-34

43. Verbeek I, Klip E (2005) Slapeloosheid. Boom, Amsterdam

44. Vijgh BH, van der Pronk V, Vugt HC, van Raymann RJ, Beun RJ (2010) A framework for sleep staging based on unobtrusive measurements. 20th Congress of the ESRS, 14-18 Sept

45. Vincent N, Lewycky S (2009) Logging on for better sleep: RCT on the effectiveness of online treatment for insomnia. Sleep 32(6):807-815

46. Watanabe T, Watanabe K (2004) Noncontact method for sleep stage estimation. IEEE Trans Biomed Eng 10(51):1735-1748 